\title{
Kablosuz güç transferinde manyetik bağlaşımdaki çevrimlerin endüktanslarının çıkarımı
}

\author{
Seyit Ahmet Sİ** \\ Elektrik-Elektronik Mühendisliği Bölümü, Mühendislik Fakültesi, Balıkesir Üniversitesi, Çă̆ış \\ Yerleşkesi, Turkiye \\ Geliş Tarihi (Recived Date): 01.10.2018 \\ Kabul Tarihi (Accepted Date): 18.10.2018
}

\section{Özet}

Kablosuz güç transferi son yıllarda üzerinde yoğun olarak çallşılan alanlardan biri durumundadur. Kablosuz güç transfer sistemlerinde aktarlan güç seviyesini ve güç transfer verimliliğini belirleyen en önemli bileşenlerden biri manyetik bağlaşımdaki bobinlerdir. Bu bobinlerin düzgün şekilde karakterize edilmeleri, verici ve alıc taraftaki devrelerin ve kompanzasyon elemanlarının seçilmesinde hayati öneme sahiptir. Bu çalışmada kablosuz güç transfer sistemlerinde kullanılan bobinlerin (coils) $\ddot{o z}$ endüktans (self-inductance) ve ortak-endüktans (mutual enductance) değerlerinin üç frakl yöntemle çıkarımları verilmektedir. Üretimi yapılmış bir artı-şekilli bobin çifti, aralarına çeşitli mesafeler konularak bir RLC metre ile ve network analizör ile ayrı ayrı ölçülmüş ve ölçüm sonuçlarından endüktans değerleri çıkarılıp karşılaştırılmıştır. Karşılaştırılan sonuçların uyumu, bu makalede özetlenen ölçüm ve yöntemlerin manyetik bağlaşımdaki bobin karakteristiklerinin çıkarımında kullanılabileceğini göstermektedir.

Anahtar kelimeler: Kablosuz güç transferi, manyetik bağlaşım, bobin.

\section{Extraction of inductances for coupled coils in wireless power transfer}

\begin{abstract}
Wireless power transfer is one of the hot research areas of electrical engineering in last decade. The most important component in a wireless power transfer system, which dictates the transferred power level and system efficiency, is magnetically coupled coil pairs. Correct characterization of the coils is extremely important for choosing or
\end{abstract}

\footnotetext{
*Seyit Ahmet SİS, seyit.sis@balikesir.edu.tr, http://orcid.org/0000-0002-3740-2391
} 
designing the right electronic circuitry and compensation compontents. In this paper, three different methods for extracting the self-and mutual-inductances of coupled coil pairs are prensented. A cross-shape coil pair is fabricated and measured using both an RLC meter and a network analyzer. Then, these three methods are utilized for extracting the inductances and the extracted inductance values are compared. The agreement in the extracted inductance values show that the presented measurements and extraction methods can be safely utilized for characterizing the magnetically coupled coils for use in wireless power transfer systems.

Keywords: Wireless power transfer, magnetic coupling, coil.

\section{Giriș}

Kablosuz güç transfer sistemleri hali hazırda elektrikli traş makineleri, elektrikli diş fırçaları gibi küçük el aletleri, yapay kalp gibi implante edilen tıbbi cihazlar ve cep telefonu, laptop gibi mobil cihazlar olmak üzere birçok uygulamada kullanılmaktadırlar [1-4]. Elektrikli traş makineleri ve diş firçaları genellikle nemli ve sslak yerlerde kullanıldığından dolayı, kablolu şarj edilmeleri/beslenmeleri elektrik çarpması riskini barındırmaktadır; dolayısıyla, kablosuz olarak şarj edilmeleri güvenlik açısından önemlidir. Öte yandan, vücuda dışarıdan içeriye kabloyu nüfuz etme durumu gerektirdiği için, vücut içerisindeki tıbbi elektronik cihazları kablo ile beslemek çoğu zaman mümkün değildir. Üstelik seçilen kablosuz güç transfer tekniğinin de vücuda zarar vermemesi gerekmektedir. Manyetik alan cisimlerle çok zayıf etkileşime girdiği için, manyetik kuplaj tekniği vücuda implante edilmiş tıbbi cihazlar içinde en uygun güç transfer yöntemidir [5]. Mobil cihazlar ve tıbbi cihazlar gibi yukarıdaki bahsedilen uygulamalar genellikle düşük güç seviyeyerinde ve genellikle $1 \mathrm{MHz}-20 \mathrm{MHz}$ aralığındaki frekanslarda çalışmaktadırlar.

Kablosuz güç transfer sistemleri için diğer önemli uygulamalarından biri de elektrikli araç şarj sistemleridir. Bunlar mobil cihazlar için olan kablosuz güç transfer sismleriyle kıyaslandığında çok daha yüksek güç sevilerinde (3 kW ve üzeri) çalışmaktadırlar. Bu seviyelere yüksek verimlilikte çıkabilmek için mobil cihazlar ve biyomedikal uygulamalara kıyasla daha düşük frekanslarda çalışılmaktadır $(20 \mathrm{kHz}-150 \mathrm{kHz})$ [6-8]. Gerek yukarıda bahsedilen düşük güçlü uygulamalarda gerekse elektrik araç şarji için yüksek güçlü kablosuz transfer sistemlerinde en önemli bileşen bobinlerdir. Bobinler Amper ve Faraday yasası esasınca verici bobin üzerinden akıtılan yüksek frekans akımın oluşturduğu manyetik alanların alıcı bobinin uçları üzerinde gerilim farkı oluşturması temelinde çalışmaktadır. Dolayısıyla bobinlerin geometrileri, sarım sayısı ve şekilleri, bobinlerin birbirleri arasındaki mesafe ve hizalanmaları yüke aktarılancak gücü doğrudan belirlemektedir. Bu nedenledirki son yıllarda özgün bobin tasarımları üzerine çok fazla çalışma yapılmıştır [9-15]. Örneğin bağlaşım sabitini artırıldı̆̆ double-D bobinleri, yine bağlaşım sabitinin artırılıp hizalanmaya karşı toleransın iyileştirildiği akı borusu (flux pipe) bobinleri konuyla ilgili literatüre sunulan çalışmaların en önemlilerindendir.

Bobinlerin gerek simulasyon gerekse ölçüm sonuçları kullanılarak elektriksel parametrelerinin düzgün şekilde çıkarılması diğer komponentlerin tasarımı yada seçimlerinde önemlidir. Bu çalışmada, bobinlerin öz ve ortak endüktans değerlerinin çıkarımları üzerine kullanılabilecek üç farklı yöntem sunulmaktadır. Bu yöntemlerden ilki RLC metre ölçümleridir. RLC metre ölçümlerinde öz endüktans $(L)$ değerleri direk ölçülebildiği için herhangibi çıkarım yapılmasına gerek yoktur. Fakat, ortak endüktans 
değerleri $(M)$ devre modeli üzerinden elde edilen çıkarım formüllerinden bulunmaktadır. İkinci yöntemde, network analizör ölçümleri sonucu elde edilen ağ parametreleri kullanılmaktadır. Üçüncü ve son yöntemde ise, sinyal üreteci ve osiloskop cihazlarının kullanımıyla $L$ ve $M$ değerleri çıkarımı yapılmaktadır.

\section{Endüktans çıkarımları}

\subsection{RLC metre ile çıkarımlar}

RLC metre ile öz endüktans değerleri direk olarak ölçülebildiği için herhangibir çıkarım söz konusu değildir. Fakat ortak endüktans $(M)$ değerinin ölçülebilmesi için uygun bir ölçüm (yada simulasyon) konfigürasyonunun belirlenmesi, ve bir bilinmeyenli denklem elde edilebilecek bir matematiksel çıkarım yapılması gerekmektedir. Manyetik olarak kuplajlanmış bobin çiftlerinin M değerlerinin RLC metre ile çıkarımı aşağıdaki adımlar kullanılarak yapılabilmektedir:

- İlk olarak her bir bobinin öz endüktans değeri ayrı ayrı ölçülüp kaydedilir.

- Daha sonra kuplajlanmış bobin çifti bir taraftan kısa devre edilirken, diğer taraftan ise RLC metre ile giriş endüktans değeri sanki tek bir izole bobin ölçülüyormuş̧̧asına ölçülür. $\mathrm{Bu}$ ölçüm konfigürasyonu aşağıda Şekil-1 de verildiği gibidir. Şekil-2 de ise, daha önceden üretilen bir artı-şekil bobinin laboratuvar ortamında Applent AT826 marka RLC metre ile yapılan ölçüm ve düzeneğinin fotoğrafı verilmektedir.

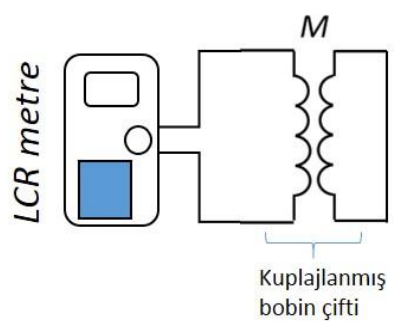

Şekil 1. RLC metre ile karşılıkı endüktans ölçüm düzeneği.

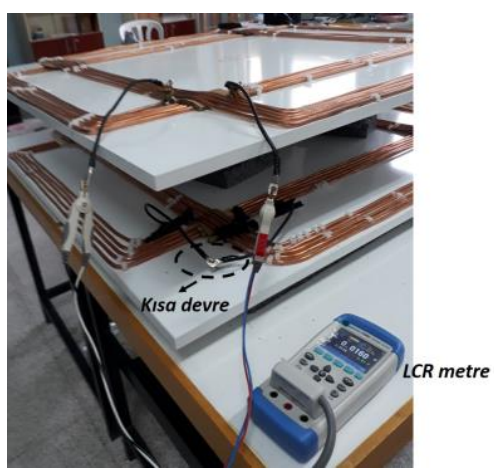

Şekil 2. RLC metre ile laboratuvarda yapılan ölçüm düzeneği. Örnek olarak üretilen bir artı şekil-bobin çifti kullanılmıştır.

Şekil-2 de ölçümü yapılan bobinler artı-şekilli bobin olup, yapısı itibariyle bağlaşım katsayısı yüksek ve yataydaki hizalanmaya toleransı da kısmen iyileştirilmiş bobin 
yapısıdır [16]. Artı-sekil bobinle ilgili detaylı çalışma sonuçları literatürde bulunmaktadır. Üretilen bu bobin yapısı $70 \mathrm{~cm}$ x $100 \mathrm{~cm}$ kenar uzunluklarında ve 5 sargıdan oluşan dikdörtgensel bobinlerin merkezleri etrafında $90{ }^{\circ}$ döndürülüp birbirleriyle elektriksel olarak paralel bağlanmasıyla elde edilmiştir. Şekil-1 deki devre modelinin bir diğer eşleniği ise endüktif-T devresidir ve aşağıda Şekil-3 de verildiği üzere gösterilmektedir.

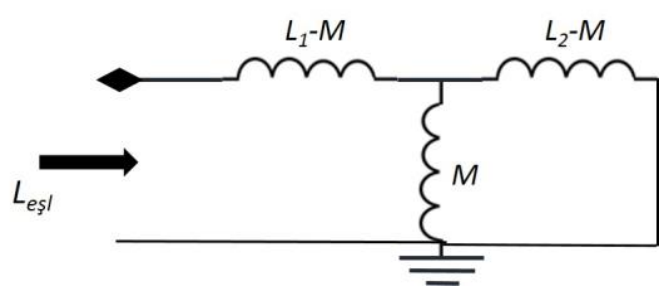

Şekil 3. Şekil-1 de verilen manyetik bağlaşımdaki çevrim çifti için endüktif-T eşlenik devresi.

- En sonunda Şekil-3 deki devre basit devre analizi teknikleri ile incelendiğinde girişinden görülen eşlenik endüktans değeri, devredeki her bir bobinin öz endüktansları $\left(L_{1}\right.$ ve $\left.L_{2}\right)$ ve ortak endüktans $(M)$ değerleri cinsinden aşağıdaki şekilde yazılabilir:

$L_{e s l}=L_{1}-M+\frac{L_{2} M-M^{2}}{L_{2}}$.

Buradan $M$ parametresi çekildiğinde ayrı ayrı ölçümü yapılan öz endüktanslar ( $L_{1}$ ve $L_{2}$ ) ve eşlenik endüktans cinsinden ifadesi aşağıdaki gibi bulunabilir:

$$
M=\sqrt{L_{2}\left(L_{1}-L_{e s l}\right)} .
$$

\subsection{Network analizör ile çıkarımlar}

Bobinlerin endüktans değeri network analizör ölçümleriyle de elde edilebilmektedir. Network analizör ölçümlerinde alıcı ve verici bobin giriş çıkışları network analizörün birinci ve ikinci portlarına bağlandığında 2-portlu S-parametre ölçümleriyle $S_{11}, S_{12}, S_{21}$, $S_{22}$ parametreleri komplex değerler olarak elde edilmektedir. Aşağıda Şekil-4 de artışekil bobin çiftinin laboratuvar ortamında FSH 8 Network+Spectrum analizör kullanılarak 2-portlu S-parametre ölçüm düzeneğinin fotoğrafı verilmektedir. Ölçülen S-parametrelerinmden aşağıda verilen dönüşüm formülleri kullanılarak Zparametrelerine dönüşüm yapılmaktadır:

$$
\begin{aligned}
& Z_{11}=Z_{0} \frac{\left(1+\mathrm{S}_{11}\right)\left(1-\mathrm{S}_{22}\right)+\mathrm{S}_{12} \mathrm{~S}_{21}}{\left(1-\mathrm{S}_{11}\right)\left(1-\mathrm{S}_{22}\right)-\mathrm{S}_{12} \mathrm{~S}_{21}} \\
& Z_{22}=Z_{0} \frac{\left(1-\mathrm{S}_{11}\right)\left(1+\mathrm{S}_{22}\right)+\mathrm{S}_{12} \mathrm{~S}_{21}}{\left(1-\mathrm{S}_{11}\right)\left(1-\mathrm{S}_{22}\right)-\mathrm{S}_{12} \mathrm{~S}_{21}}
\end{aligned}
$$




$$
\begin{aligned}
& Z_{12}=Z_{0} \frac{2 S_{12}}{\left(1-S_{11}\right)\left(1-S_{22}\right)-S_{12} S_{21}} \\
& Z_{21}=Z_{0} \frac{2 S_{21}}{\left(1-S_{11}\right)\left(1-S_{22}\right)-S_{12} S_{21}}
\end{aligned}
$$

(3)-(6) da denklemlerde $Z_{0}$ sistem empedansı olup bu ölçümlerde $50 \Omega$ dur.

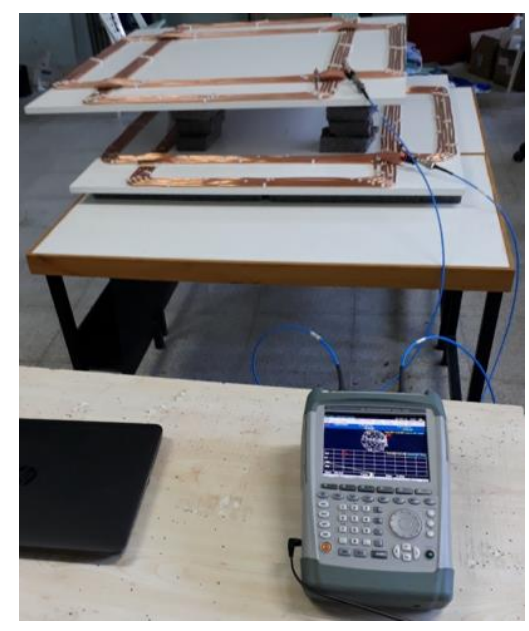

Şekil 4. Laboratuvar ortamında 2-portlu S-parametre ölçüm düzeneği.

Z-parametrelerinden $L_{1}, L_{2}$ ve $M$ değerlerinin çıkarımı aşağıda Şekil-5 de verilen endüktif-T devresinin Z- parametrelerinin hesaplanmasıyla gerçekleştirilebilir. Aşağıdaki devrenin analiz edilmesiyle elde edilen Z-parametreleri (7)-(9) da verilmektedir.

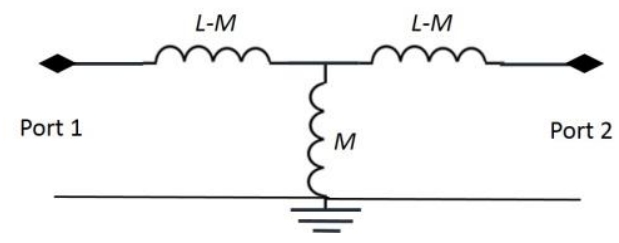

Şekil 5. Manyetik bağlaşımdaki endüktif bobin çiftinin 2-portlu eşlenik endüktif-T devre modeli.

$$
\begin{aligned}
& Z_{11}=j \omega\left(L_{1}-M\right)+j \omega M \\
& Z_{22}=j \omega\left(L_{2}-M\right)+j \omega M \\
& Z_{12}=Z_{21}=j \omega M
\end{aligned}
$$

Buradan $L$ ve $M$ değerleri ölçümlerden elde edilen Z-parametrelerinden aşağıdaki şekilde çıkarilabilir. 


$$
\begin{aligned}
& L_{1}=\frac{\operatorname{imag}\left(\mathrm{Z}_{11}\right)}{2 \pi f} \\
& L_{2}=\frac{\operatorname{imag}\left(\mathrm{Z}_{22}\right)}{2 \pi f} \\
& M=\frac{\operatorname{imag}\left(\mathrm{Z}_{21}\right)}{2 \pi f}
\end{aligned}
$$

\subsection{Sinyal üreteci ve osiloskopla çıkarımlar}

$\mathrm{Bu}$ çalışmada üretimi yapılan artı-şekil bobinlerin karşılıklı endüktans değerieri sinyal üreteci ve osiloskop kullanılarak da çıkarılmış ve yukarıda anlatılan RLC metre ve network analizör ölçümlerinden yapılan çıkarımlarla karşılaştırılmıştır. Sinyal üreteci manyetik olarak bağlaşımdaki bobin çiftinin verici tarafından girişine bağlanmakta olup alıcının çıkışı açık devre olarak bırakılmaktadır. Aşağıda Şekil-6 da ölçüm konfigürasyonu verilmektedir.

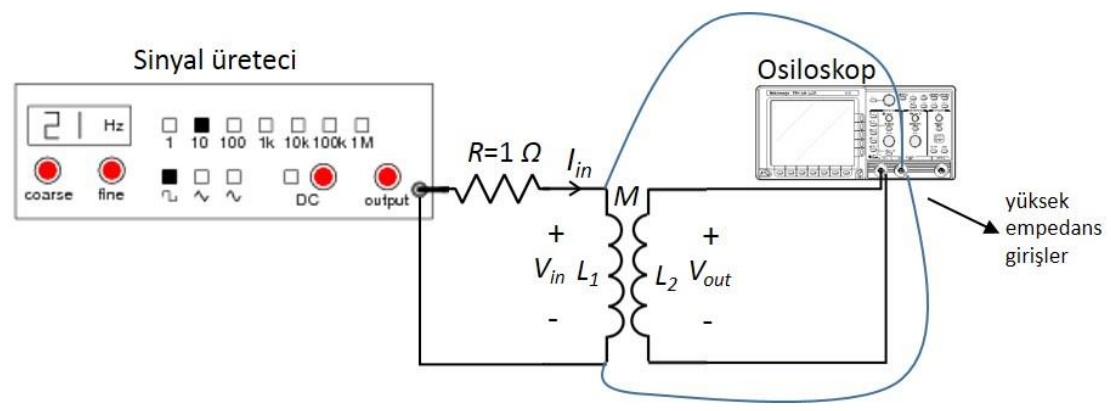

Şekil 6. Sinyal üreteci ve osiloskop kullanılarak endüktans değerleri için ölçüm düzeneği.

Şekil-6 daki düzenekte, sinyal üretecinden bir sinusoidal sinyal verilmekte ve giriş akımı $\left(I_{\text {in }}\right)$, giriş voltajı $\left(V_{\text {in }}\right)$ ve çıkış voltajı $\left(V_{\text {out }}\right)$ ölçülmektedir. Sinusoidal giriş akımı, $1 \Omega$ luk $R$ direnci üzerindeki gerilimin osiloskop üzerinde ölçülmesiyle elde edilmektedir. Bu ölçümler sonucu $L_{l}$ ve $M$ değerleri sırasıyla (13) ve (14) de verildiği üzere çıkarılabilir. $L_{2}$ değeri de giriş ve çıkışların yerleri değiştirilerek aynı ölçüm ve (13) ü kullanılarak elde edilebilir.

$$
\begin{aligned}
& L_{1}=\frac{V_{\text {in }}}{2 \pi f I_{\text {in }}} . \\
& M=\frac{V_{\text {out }}}{2 \pi f I_{\text {in }}} .
\end{aligned}
$$

\section{Sonuçlar ve tartışma}

Yukarıda bölüm 2.1, 2.2 ve 2.3 deki çıkarım teknikleri ilgili uygun ölçüm sonuçlarına uygulanarak çıkarımlar yapılmıştır. Bu bölümde bu çıkarım sonuçları verilmekte ve sonuçlar yorumlanmaktadır. Aşağıda Tablo 1. de bölüm 2.1 de kullanılan teknik sonucu 
çıkarımı yapılan $L_{1}, L_{2}$ ve $M$ değerleri RLC metrede varolan frekans adımlarında verilmektedir.

Tablo 1. Bölüm 2.1 de RLC metre ölçümlerinden elde edilen $L$ ve $M$ değerleri.

\begin{tabular}{|c|c|c|c|c|c|}
\hline & $L_{1}(\mu)$ & $L_{2}(\mu)$ & $\begin{array}{c}M(\mu H), d=14 \\
\mathrm{~cm}\end{array}$ & $\begin{array}{c}M(\mu H), \\
d=17.5 \mathrm{~cm}\end{array}$ & $\begin{array}{c}M(\mu H), \\
d=21 \mathrm{~cm}\end{array}$ \\
\hline $100 \mathrm{~Hz}$ & 37 & 38 & 15.1 & 13.8 & 10.7 \\
\hline $120 \mathrm{~Hz}$ & 37 & 38 & 16.3 & 13.8 & 10.7 \\
\hline $1 \mathrm{kHz}$ & 34.5 & 34.7 & 14.7 & 12.4 & 10.2 \\
\hline $10 \mathrm{kHz}$ & 33.7 & 33.3 & 14 & 11.6 & 9.35 \\
\hline $100 \mathrm{kHz}$ & 33.4 & 32.7 & 13.7 & 11.2 & 8.91 \\
\hline
\end{tabular}

Aşağıda Şekil- 7 ve Şekil-8 de bölüm 2.2 de anlatılan network analizör ölçümlerinde çıkarımı yapılmış L ve $\mathrm{M}$ değerlerinin $150 \mathrm{kHz}-1 \mathrm{MHz}$ arası frekansa göre değişim grafikleri verilmektedir.

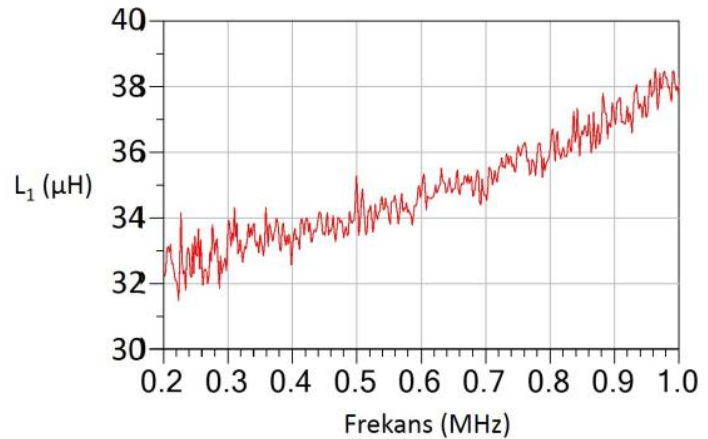

(a)

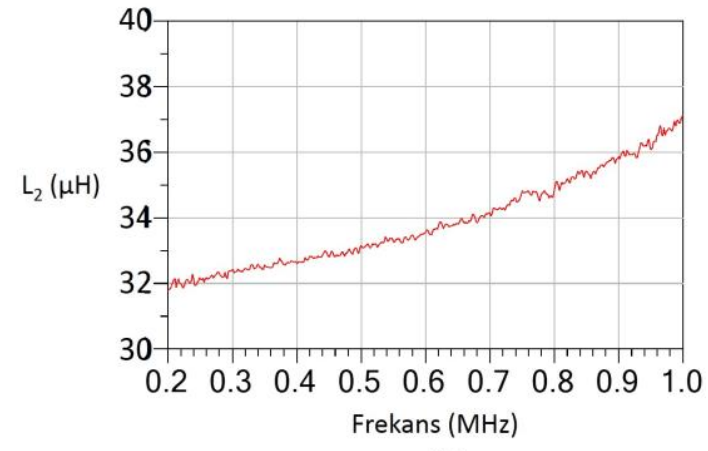

(b)

Şekil 7. Network analizör ölçümlerinden çıkarımı yapılan öz endüktans değeleri. Network analizörde S-parametreleri $1 \mathrm{MHz}$ e kadar ölçüldüğü için çıkarımlarda bu frekansa kadar yapılmıştır. 


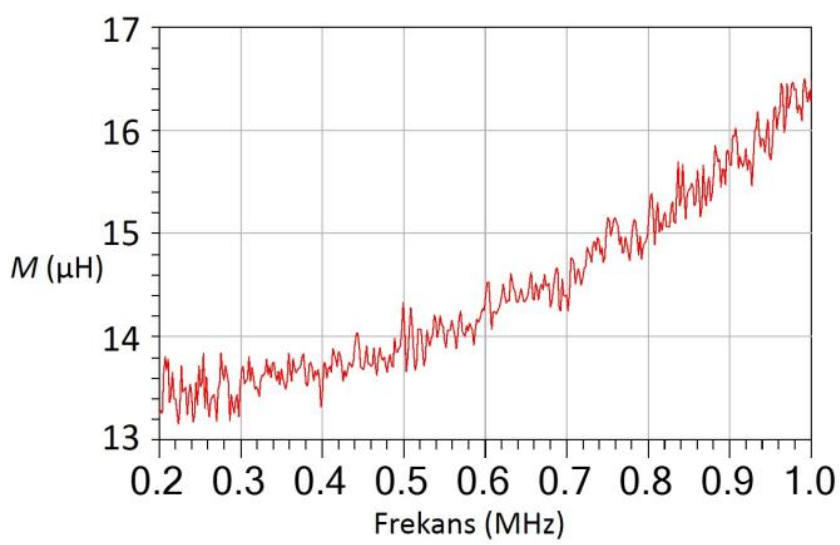

Şekil 8. Network analizör ölçümlerinden çıkarımı yapılan $14 \mathrm{~cm}$ mesafe için ortak endüktans değeleri. Network analizörde S-parametreleri $1 \mathrm{MHz}$ e kadar ölçüldüğü için çıkarımlarda bu frekansa kadar yapılmıştır.

Son olarak aşağıda Tablo 2. de bölüm 2.3 de anlatılan ve osiloskop ve sinyal üreteci kullanılarak yapılan ölçümlerden çıkarılan öz endüktans ve karşılıklı endüktans değerleri verilmektedir.

Tablo 2. Bölüm 2.3 de sinyal üreteci ve osiloskop ölçümlerinden çıkarımı yapılan $L$ ve $M$ değerleri.

\begin{tabular}{|c|c|c|c|c|}
\hline & $L_{1}(\mu)$ & $L_{2}(\mu)$ & $\begin{array}{c}M(\mu H), d=14 \\
\mathrm{~cm}\end{array}$ & $\begin{array}{c}M(\mu H), \\
d=21 \mathrm{~cm}\end{array}$ \\
\hline $150 \mathrm{kHz}$ & 30 & 30.1 & 15 & 10.8 \\
\hline $300 \mathrm{kHz}$ & 33 & 33.9 & 15.8 & 11.5 \\
\hline $500 \mathrm{kHz}$ & 38 & 39 & 17 & 13.2 \\
\hline
\end{tabular}

Tablo-1, Şekil-7, Şekil-8 ve Tablo-2 de verilen sonuçlara bakıldığında, çıkarımların yapıldığı freans aralıkla birbirlerinden farklı olmalarına rağmen çıkarımı yapılan $L$ ve $M$ değerleri oldukça uyumludur. Frekans arttıkça çıkarımı yapılan L ve M değerlerinin doğruluk payı hesaba katılmayan parazit etkilerden dolayı düşecektir. Örneğin, çok sargılı bobinlerde sargılar arası parazit kapasitans etkisi yüksek frekanslarda daha fazladır. Yine, frekans arttıkça deri etkisinden dolayı kayıp dirençleri artacaktır. Bu çalışmada bu parazit etkiler hesaba katılmamıştır. Lakin çalışmada özetlenen çıkarım yöntemleri özellike kablosuz güç transferi konusunda çalışmaya yeni başlayan araştırmacılar için bobin karakterizasyonunda yardımcı olacak yöntemleri vermektedir.

\section{Kaynaklar}

[1] Fernandez, C., Garcia, O., Prieto, R., Cobos, J. A., Uceda, J., Overview of different alternatives for the contact-less transmission of energy, IECON 02, 2, 1318-1323, (2002).

[2] Soma, Mani., Galbraith, D.C., White, R.L., Radio-frequency coils in implantable devices: Misalignment analysis and design procedure, IEEE transactions on biomedical engineering, 4, 276-282, (1987). 
[3] Xiaolin, M., Sun ,H., Wireless power transfer: Survey and roadmap, Vehicular Technology Conference (VTC Spring), 1-5, (2015).

[4] Xie, L., Shi, Y., Hou, Y.T., Lou, A., Wireless power transfer and applications to sensor networks, IEEE Wireless Communications, 20(4),140-145, (2013).

[5] Yeh, J.C., Huang, K.L., Hsiao, Y.C., Hsu, Y.H., Lin, Y.H., Lou, S.L., Lee, T.H., A rat model of thrombosis in common carotid artery induced by implantable wireless light-emitting diode device, BioMed research international, (2014).

[6] Siqi, L., Mi, C.C., Wireless power transfer for electric vehicle applications, IEEE journal of emerging and selected topics in power electronics, 3(1), 4-17, (2015).

[7] Covic, G. A., Boys, J.T., Modern trends in inductive power transfer for transportation applications, IEEE Journal of Emerging and Selected topics in power electronics, 1(1), 28-41, (2013).

[8] Vilathgamuwa, D. M., Sampath J. P. K, Wireless power transfer (WPT) for electric vehicles (EVS) - Present and future trends, Plug in electric vehicles in smart grids, Springer, Singapore, 33-60, (2015).

[9] Budhia, M., Covic, G., Boys, J., A new IPT magnetic coupler for electric vehicle charging systems, In Proceedings of the IECON 2010-36th Annual Conference on IEEE Industrial Electronics Society, Glendale, AZ, USA, 2487-2492, (2010).

[10] Budhia, M., Boys, J.T., Covic, G.A., Huang, C.Y., Development of a singlesided flux magnetic coupler for electric vehicle IPT charging systems, IEEE Trans. Ind. Electron, 60, 318-328, (2013).

[11] Nguyen, T.D., Li, S., Li, W., Mi, C.C., Feasibility study on bipolar pads for efficient wireless power chargers, IEEE Applied Power Electronics Conference and Exposition-APEC 2014, Fort Worth, TX, USA, 1676-1682, (2014).

[12] Deng, J., Li, W., Nguyen, T.D., Li, S., Mi, C.C., Compact and efficient bipolar coupler for wireless power chargers: design and analysis, IEEE Trans. Power Electron, 30, 6130-6140, (2015)

[13] Wei, Q., Guo, W., Sun, X., Wang, G., Zhao, X., Li, F., Li, Y., A new type of ipt system with large lateral tolerance and its circuit analysis. International Conference on Connected Vehicles and Expo (ICCVE), Beijing, China, 311315, (2012).

[14] Li, Y., Lin, T., Mai, R., Huang, L., He, Z., Compact double-sided decoupled coils-based wpt systems for high-power applications: analysis, design, and experimental verification, IEEE Trans. Transp. Electrification, 4, 64-75, (2018).

[15] Jonah, O., Georgakopoulos, S.V., Tentzeris, M.M., Orientation insensitive power transfer by magnetic resonance for mobile devices, IEEE Wireless Power Transfer (WPT), Perugia, Italy, 5-8, (2013).

[16] Sis, S.A., Orta, E., A cross-shape coil structure for use in wireless power applications, Energies, 11, 1094, (2018). 